\title{
Effect of 3D magnetic perturbations on the plasma rotation in ASDEX Upgrade
}

\author{
A F Martitsch ${ }^{1}$, S V Kasilov ${ }^{1,2}$, W Kernbichler ${ }^{1}$, G Kapper ${ }^{1}$, \\ C G Albert ${ }^{1}$, M F Heyn ${ }^{1}$, H M Smith ${ }^{3}$, E Strumberger ${ }^{4}$, \\ S Fietz ${ }^{4}$, W Suttrop ${ }^{4}$, M Landreman ${ }^{5}$, the ASDEX Upgrade \\ Team $^{4}$ and the EUROfusion MST1 Team $\ddagger$ \\ ${ }^{1}$ Fusion@ÖAW, Institut für Theoretische Physik - Computational Physics, TU Graz, \\ Petersgasse 16, A-8010 Graz, Austria \\ ${ }^{2}$ Institute of Plasma Physics, National Science Center "Kharkov Institute of Physics \\ and Technology", Ul. Akademicheskaya 1, 61108 Kharkov, Ukraine \\ ${ }^{3}$ Max-Planck Institut für Plasmaphysik, D-17491 Greifswald, Germany \\ ${ }^{4}$ Max-Planck Institut für Plasmaphysik, D-85748 Garching, Germany \\ ${ }^{5}$ Institute for Research in Electronics and Applied Physics, University of Maryland, \\ College Park, Maryland 20742, USA
}

E-mail: martitsch@tugraz.at

December 2015

\begin{abstract}
The toroidal torque due to the non-resonant interaction with external magnetic perturbations ( $\mathrm{TF}$ ripple and perturbations from ELM mitigation coils) in ASDEX Upgrade is modelled with help of the NEO-2 and SFINCS codes and compared to semi-analytical models. It is shown that almost all non-axisymmetric transport regimes contributing to neoclassical toroidal viscosity (NTV) are realized within a single discharge at different radial positions. The NTV torque is obtained to be roughly a quarter of the NBI torque. This indicates the presence of other important momentum sources. The role of these momentum sources and possible integral torque balance measurements are briefly discussed.
\end{abstract}

\section{Introduction}

Stability and transport of tokamak plasmas are strongly influenced by toroidal plasma rotation. The knowledge of various mechanisms and corresponding torques driving the toroidal plasma rotation is therefore crucial for operation control of these fusion experiments. Dedicated experimental studies at NSTX [1], DIII-D [2] and JET [3, 4] have shown a strong dependence of the plasma rotation on non-axisymmetric magnetic perturbations (e.g., from coils for mitigation of edge localized modes (ELMs), from toroidal field (TF) coil ripple and from error fields). The observed changes in plasma rotation were in agreement with theoretical predictions for the torque produced by $\ddagger$ See http://www.euro-fusionscipub.org/mst1 
non-resonant non-axisymmetric magnetic perturbations, which are based on analytical and semi-analytical approaches $[5,6,7]$. The non-resonant torque produced by such perturbations is often expressed through a viscous force, terming the phenomenon also as neoclassical toroidal plasma viscosity (NTV). In this paper the NTV torque is evaluated numerically for ASDEX Upgrade equilibria using the drift kinetic equation (DKE) solver NEO-2 [8, 9] and the results are compared to analytical models [5, 10] and a semi-analytical approach based on Hamiltonian theory [11], as well as to results from the DKE solver SFINCS [12].

Analytical and semi-analytical approaches presently used for the evaluation of the NTV torque [5, 6, 7] make simplifying assumptions concerning geometry and collision operators. A numerical approach without such simplifications is provided by the quasilinear version of the code NEO-2 [8]. In this code, the only assumption simplifying the general neoclassical ansatz for non-axisymmetric tori is that perturbations of the magnetic field are small enough such that the particle motion within perturbed flux surfaces is only weakly affected by the perturbation field. This reduces the "nonlinear" 4D problem, where all relevant toroidal Fourier modes of the perturbation field simultaniously affect the particle motion, to a set of uncoupled 3D problems. Those are solved for each toroidal Fourier mode separately and finally result in independent contributions to the torque. Such a quasilinear approach is well justified in many circumstances but might be only marginally justified for high toroidal mode numbers of the perturbation. In a previous study [8], the quasilinear version of the code NEO-2 has been benchmarked for a simplified tokamak geometry against various analytical models [5, 13, 14, 15, 16, 10], as well as the nonlinear codes DKES [17] and NEO [18]. In this paper, also the DKE solver SFINCS [12] is used for benchmarking of the ion contribution to the torque. The code SFINCS is not limited to small values of the perturbation amplitude because it solves the nonlinear problem pertinent to neoclassical stellarator transport. Computationally, this is a much more demanding task than solving the quasilinear problem. In contrast to the DKES code, which solves the reduced monoenergetic problem, SFINCS as NEO-2 uses the full linearized Coulomb collision operator.

A set of perturbed ASDEX Upgrade equilibria studied here has been computed by the ideal MHD equilibrium solver NEMEC [19, 20]. These equilibria include both, perturbations from the TF ripple and from ELM mitigation coils with different distribution of current values resulting in different perturbation field spectra in the ASDEX Upgrade shot \#30835. Due to the strong shielding of resonant magnetic perturbations (RMPs) by plasma response currents in AUG [21], magnetic fields computed within ideal MHD theory, where RMPs are shielded perfectly, provide a good approximation in a major part of the plasma volume except for narrow resonant layers around resonant rational flux surfaces.

Here, in order to identify various NTV regimes of importance for ASDEX Upgrade, comparison of numerical results from NEO-2 and SFINCS with several analytical models and additional parameter scans have been undertaken. In addition, results for the 
integral torque are compared to the torque resulting from neutral beam injection (NBI) computed by the code NUBEAM [22] and the overall torque balance is discussed.

The structure of the paper is as follows. In Section 2 the toroidal moment conservation equation and its simplified forms are introduced and basic definitions are given, e.g., expressions for the rotation velocity components and toroidal torque density in terms of plasma parameters and neoclassical transport coefficients. In Section 3 the NTV torque profiles computed by NEO-2 and SFINCS are shown for ASDEX Upgrade equilibria, as well as a comparison to analytical and semi-analytical models is presented. In Section 4 additional momentum sources, which are not taken into account here, are discussed in order to stimulate further experiments and simulations, and finally in Section 5 the results are summarized.

\section{Toroidal momentum conservation and neoclassical toroidal viscosity}

In a tokamak plasma, charged particles and neutrals together with the electromagnetic field are represented by a coupled system, which can be characterized by the exact conservation law of the total toroidal momentum of particles and of the electromagnetic field. In a covariant notation this conservation law is given as (see, e.g., [8] for its toroidally averaged form),

$$
\frac{\partial}{\partial t} P_{\varphi}+\left(\frac{\partial x^{i}}{\partial t}\right)_{\mathbf{r}} \frac{\partial}{\partial x^{i}} P_{\varphi}+\frac{1}{\sqrt{g}} \frac{\partial}{\partial x^{i}} \sqrt{g} \Pi_{\varphi}^{i}=0,
$$

where $\sqrt{g}$ is the metric determinant and $x^{i}$ are some (generally time dependent) coordinates with rotational symmetry over the toroidal angle $\varphi$, such as cylindrical coordinates $(R, \varphi, Z)$ or flux coordinates $(r, \vartheta, \varphi)$ associated with the unperturbed axisymmetric field, and $P_{\varphi}=\mathbf{P} \cdot \partial \mathbf{r} / \partial \varphi$ and $\Pi_{\varphi}^{i}=(\partial \mathbf{r} / \partial \varphi) \cdot \boldsymbol{\Pi} \cdot \nabla x^{i}$, are the toroidal co-variant components of the momentum density vector and the total stress tensor of particles and electromagnetic field respectively given by

$$
\mathbf{P}=\sum_{\alpha} \mathbf{P}_{(\alpha)}+\frac{1}{c^{2}} \mathbf{S}, \quad \boldsymbol{\Pi}=\sum_{\alpha} \boldsymbol{\Pi}_{(\alpha)}-\boldsymbol{\sigma} .
$$

Here,

$$
\mathbf{P}_{(\alpha)}=m_{\alpha} n_{\alpha} \mathbf{V}_{\alpha}, \quad \boldsymbol{\Pi}_{(\alpha)}=m_{\alpha} n_{\alpha} \mathbf{V}_{\alpha} \mathbf{V}_{\alpha}+p_{\alpha} \mathbf{I}+\boldsymbol{\pi}_{\alpha}
$$

are the momentum density vector and the total stress tensor of particle species $\alpha$, respectively, with pertinent mass $m_{\alpha}$, density $n_{\alpha}$, scalar pressure $p_{\alpha}$, and viscous stress tensor $\boldsymbol{\pi}_{\alpha}$. Pointing flux $\mathbf{S}$ and Maxwell stress tensor $\boldsymbol{\sigma}$ are respectively defined as

$$
\mathbf{S}=\frac{c}{4 \pi} \mathbf{E} \times \mathbf{B}, \quad \boldsymbol{\sigma}=\frac{1}{4 \pi}\left(\mathbf{E} \mathbf{E}-\frac{E^{2}}{2} \mathbf{I}+\mathbf{B} \mathbf{B}-\frac{B^{2}}{2} \mathbf{I}\right) .
$$

In the main plasma volume neoclassical "flux surface" averaging of (1) over unperturbed flux surfaces results in a one dimensional conservation law,

$$
\frac{1}{S} \frac{\partial}{\partial t} S\left\langle P_{\varphi}\right\rangle+\frac{1}{S} \frac{\partial}{\partial r} S\left\langle\Pi_{\varphi}^{r}\right\rangle=0
$$


where $\langle\ldots\rangle$ denotes the average, $r$ is a flux surface label (effective radius) fixed by the condition $\langle|\nabla r|\rangle=1$, and $S$ is the (generally time dependent) flux surface area.

The exact equations (1) and (5) contain no volume source density. This means that the integral total momentum within the vacuum vessel can only be driven by sources located at the walls or outside the vessel. Since the Pointing flux is usually negligibly small, the toroidal momentum is approximately the same as the kinematic toroidal momentum of plasma particles and neutrals. However, the contribution of the electromagnetic field, $\boldsymbol{\sigma}$, to the total momentum flux density $\left\langle\Pi_{\varphi}^{r}\right\rangle$ is as important as the contributions by charged particles and neutrals, $\boldsymbol{\Pi}_{(\alpha)}$. In order to make equation (5) of practical use within a local 1D balance description, the nonlocal fluxes, which are not fully determined by local plasma parameters and a limited number of their derivatives, should be excluded from the total momentum flux $\left\langle\Pi_{\varphi}^{r}\right\rangle$ and turned into momentum sources (torque densities), which are described outside the closed set of balance equations. E.g., excluding the contribution of neutral particles, $\alpha=\mathrm{n}$, produced by NBI from the l.h.s. of (5), the pertinent torque density is obtained as

$$
T_{\varphi}^{\mathrm{NBI}}=-\frac{1}{S} \frac{\partial}{\partial t} S P_{(\mathrm{n}) \varphi}-\frac{1}{S} \frac{\partial}{\partial r} S\left\langle\Pi_{(\mathrm{n}) \varphi}^{r}\right\rangle .
$$

Formally the contributions from all external non-axisymmetric electromagnetic perturbations, including besides the static or slowly varying magnetic perturbations also the contribution from RF heating and current drive, can be separated into the source term $T_{\varphi}^{\mathrm{NA}}$. Generally, this separation is not so straightforward as (6), see, e.g., [8] for the case of non-resonant magnetic perturbations, and may even not always be meaningful in a general case (see discussion in Section 4). Thus, equation (5) turns into

$$
\frac{1}{S} \frac{\partial}{\partial t} S \sum_{\alpha} m_{\alpha}\left\langle g_{\varphi \varphi} n_{\alpha} V_{\alpha}^{\varphi}\right\rangle+\frac{1}{S} \frac{\partial}{\partial r} S\left\langle\Pi_{[\mathrm{in}] \varphi}^{r}\right\rangle=T_{\varphi}^{\mathrm{NBI}}+T_{\varphi}^{\mathrm{NA}},
$$

where $\alpha$ denotes only plasma particles. The radial component of the total stress $\left\langle\Pi_{[\mathrm{in}] \varphi}^{r}\right\rangle$ (flux surface averaged radial flux density of the toroidal momentum) is the sum of the total stress from intrinsic turbulent modes (anomalous momentum flux density) and total axisymmetric stress including the contribution from the polarization current and a small contribution of axisymmetric neoclassical shear viscosity. In case of non-resonant external magnetic perturbations, $T_{\varphi}^{\mathrm{NA}}$ is directly linked through the flux-force relation to the non-ambipolar neoclassical particle flux densities $\Gamma_{\alpha}^{\mathrm{NA}}$ driven by these perturbations in a stationary radial electric field (see, e.g. $[5,8]$ ),

$$
T_{\varphi}^{\mathrm{NA}}=-\frac{1}{c} \sqrt{g} B^{\vartheta} \sum_{\alpha} e_{\alpha} \Gamma_{\alpha}^{\mathrm{NA}}
$$

Here, $c$ is the speed of light, $e_{\alpha}$ is the charge of species $\alpha$, and $B^{\vartheta}$ is the contra-variant magnetic field component linked to the poloidal flux $\psi_{\text {pol }}$ by $\sqrt{g} B^{\vartheta}=\partial \psi_{\text {pol }} / \partial r$. Torque produced by these thermal particle fluxes is called NTV torque. In presence of suprathermal particle losses, the torque density (8) should include also the flux of these fast particles (see, e.g., [23, 24]). However, this type of flux cannot be described by the local neoclassical ansatz. 
The non-ambipolar neoclassical particle flux densities are expressed through transport coefficients $D_{i j}^{\mathrm{NA}}$ and thermodynamic forces $A_{j}$,

$$
\Gamma_{\alpha}^{\mathrm{NA}}=-n_{\alpha}\left(D_{11}^{\mathrm{NA}} A_{1}+D_{12}^{\mathrm{NA}} A_{2}\right)
$$

where the thermodynamic forces are specified by

$$
\begin{aligned}
& A_{1}=\frac{1}{n_{\alpha}} \frac{\partial n_{\alpha}}{\partial r}-\frac{e_{\alpha} E_{r}}{T_{\alpha}}-\frac{3}{2 T_{\alpha}} \frac{\partial T_{\alpha}}{\partial r}, \\
& A_{2}=\frac{1}{T_{\alpha}} \frac{\partial T_{\alpha}}{\partial r}
\end{aligned}
$$

with $T_{\alpha}$ and $E_{r}$ being $\alpha$ species temperature and radial electric field, respectively. Thus, equations (8) and (9) reduce the problem to the evaluation of diffusion coefficients $D_{i j}^{\mathrm{NA}}$, which are computed here numerically by NEO-2, SFINCS, and the Hamiltonian approach [11] using the perturbed equilibrium magnetic fields from the NEMEC code represented in Boozer coordinates $(r, \vartheta, \varphi)$. For the perturbed equilibria, these variables correspond to the perturbed magnetic field and are different from flux variables used in equations (1)-(7). In particular, $\varphi$ is not an exact symmetry variable anymore. This difference, however, is small for weakly perturbed equilibria and can be ignored in (7) (see [8]). For evaluation of the analytical expressions of Shaing [5] magnetic fields have been converted from Boozer coordinates to Hamada coordinates. The radial electric field profile required for the forces (10) is calculated here from the toroidal rotation frequency of ions via the relation

$$
\begin{aligned}
V^{\varphi} & =\frac{c}{\sqrt{g} B^{\vartheta}}\left(E_{r}-\frac{1}{e_{\mathrm{i}} n_{\mathrm{i}}} \frac{\partial\left(n_{\mathrm{i}} T_{\mathrm{i}}\right)}{\partial r}\right)+q V^{\vartheta}, \\
V^{\vartheta} & =\frac{c k B_{\varphi}}{e_{\mathrm{i}} \sqrt{g}\left\langle B^{2}\right\rangle} \frac{\partial T_{\mathrm{i}}}{\partial r}
\end{aligned}
$$

where $q$ is the safety factor. The coefficient $k=5 / 2-D_{32} / D_{31}$ is determined by the parallel ion flow obtained from the NEO-2 solution for the unperturbed, axisymmetric problem,

$$
\begin{aligned}
\left\langle V_{\| \mathrm{i}} B\right\rangle & =B_{\varphi}\left(V^{\varphi}-q V^{\vartheta}\right)+\frac{c k B_{\varphi}}{e_{\mathrm{i}} \sqrt{g} B^{\vartheta}} \frac{\partial T_{\mathrm{i}}}{\partial r} \\
& =-\left(D_{31} A_{1}+D_{32} A_{2}\right) .
\end{aligned}
$$

\section{NTV torque in ASDEX-Upgrade}

Evaluation of the NTV torque is performed here for a set of ASDEX Upgrade equilibria based on the shot \#30835 $\left(B_{t}=-1.794 \mathrm{~T}, I_{p}=0.8 \mathrm{MA}, P_{\text {heat }}=9.753 \mathrm{MW}, \nu^{\star}=0.03\right.$, $\kappa=1.753, \delta_{o}=0.151, \delta_{u}=0.511$, H-mode). ASDEX Upgrade is equipped with 16

ELM mitigation coils, which form an upper and a lower ring each consisting of eight coils [25]. This setup allows for some control of the poloidal mode spectrum by varying the toroidal phase shift between the upper and lower coils $\Delta \phi_{\mathrm{ul}}$, which is also termed as varying the coil polarity. The NTV torque is computed here for the experimentally realized ELM mitigation coil polarity $\Delta \phi_{\mathrm{ul}}=90^{\circ}$ where good ELM mitigation has 
been achieved, as well as for a few simulated equilibria with other coil polarities. A simple (pure deuterium) plasma is assumed in this analysis. This assumption only weakly overestimates the ion density in case of high $Z$ impurities and $Z_{\text {eff }} \sim 1.7$. In Figure 1 the experimentally measured profiles of density $n_{\mathrm{e}}$, temperatures $T_{\mathrm{i}}$ and $T_{\mathrm{e}}$, toroidal ion rotation frequency $V^{\varphi}$, collisionality parameter $\nu^{\star}=2 \nu q R_{0} v_{T}^{-1}$, and the resulting toroidal Mach number of the $\mathbf{E} \times \mathbf{B}$ rotation $M_{t}=c R_{0} E_{r}\left(v_{T} \sqrt{g} B^{\vartheta}\right)^{-1}$ as well as the safety factor for the corresponding shot are shown as functions of the normalized poloidal radius $\rho_{\text {pol }}=\left(\psi_{\text {pol }} / \psi_{\text {pol }}^{a}\right)^{1 / 2}$. Here, $\nu=16 \sqrt{\pi} n_{\alpha} e_{\alpha}^{4} \Lambda\left(3 m_{\alpha}^{2} v_{T}^{3}\right)^{-1}, \Lambda$ is the Coulomb logarithm, $v_{T}=\left(2 T_{\alpha} / m_{\alpha}\right)^{1 / 2}, R_{0}$ is the mean major radius value at a given flux surface, $\psi_{\text {pol }}=0$ on the magnetic axis and $\psi_{\text {pol }}^{a}$ is the poloidal flux value at the separatrix. The radial electric fields obtained from NEO-2 agree perfectly with those from SFINCS computations (not shown in this figure). Within this modeling effort of
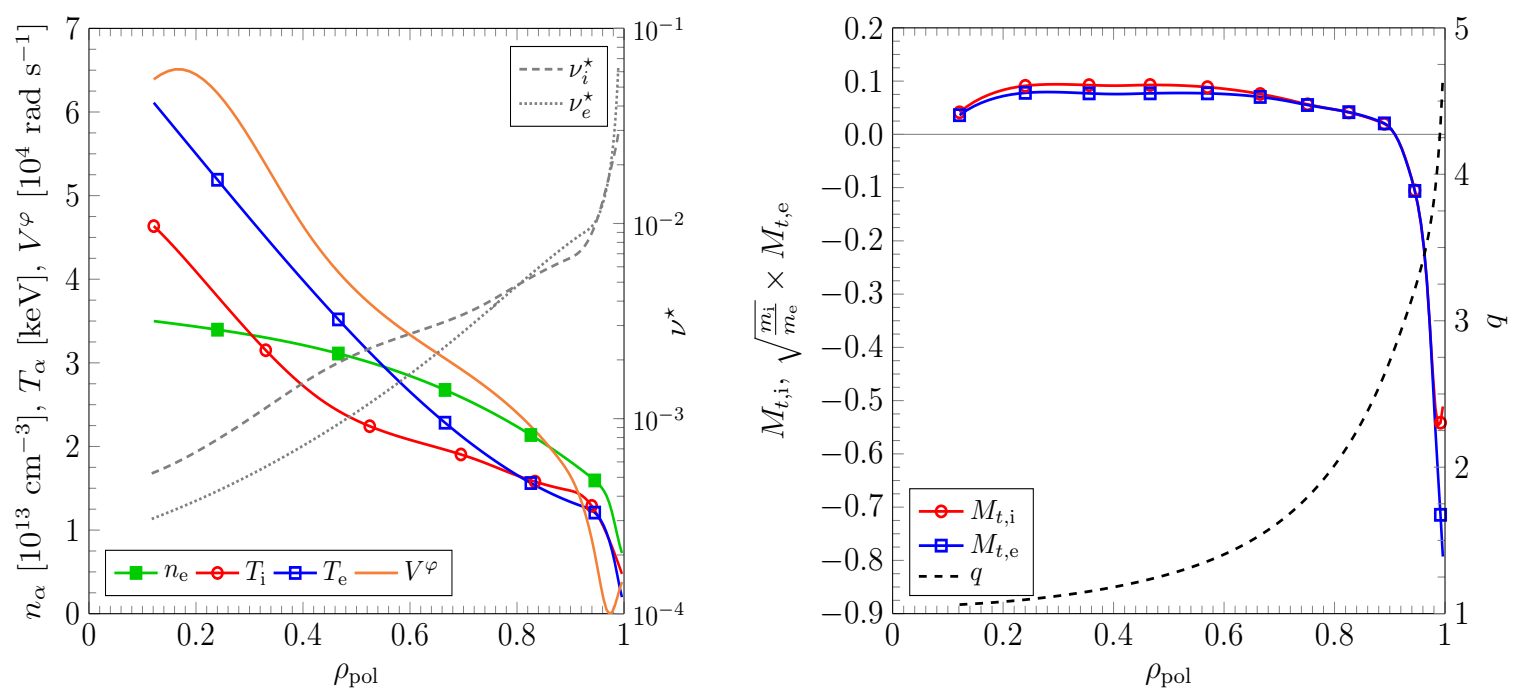

Figure 1. Radial profiles of density, temperatures, toroidal rotation frequency, collisionalities (left), toroidal Mach number and safety factor (right) for ASDEX Upgrade shot \#30835 (with ELM mitigation coils switched on).

the NTV torque, magnetic perturbations due to both TF ripple (toroidal mode number $n=16)$ and ELM mitigation coils with various coil polarities in this shot $(n=2$ with a minor contribution from $n=6$ ), are studied (see Figure 2).

In Figure 3 radial profiles and scans over the normalized perpendicular adiabatic invariant $\eta=v_{\perp}^{2}\left(v^{2} B\right)^{-1}$ of the ion $\mathbf{E} \times \mathbf{B}$ drift frequency $\Omega_{t E}=M_{t} v_{T} R_{0}^{-1}$, bounceaveraged magnetic drift frequency $\left\langle\Omega_{t B}\right\rangle_{\mathrm{b}}$ (see definition in (16)) and bounce frequency $\omega_{\mathrm{b}}$ are shown for various $\eta$-values and radial positions, respectively. Resonances between the different frequencies lead to the formation of resonant transport regimes, which are described by asymptotical formulas in the collisionless limit $[10,5,16,6]$. The resonance condition for the superbanana-plateau (sb-p) regime $[26,11]$ is given by $\Omega_{t E}+\left\langle\Omega_{t B}\right\rangle_{\mathrm{b}}=0$, whereas for drift-orbit resonances $[10,16,6,11]$ the condition $m_{\vartheta} \omega_{\mathrm{b}}+n\left(\Omega_{t E}+\left\langle\Omega_{t B}\right\rangle_{\mathrm{b}}\right)=0$ must be fulfilled for trapped particles. Here, the bounce frequency mode number $m_{\vartheta}$ can take any positive or negative integer value. It can be seen that the sb-p resonance 
condition is fulfilled in the inner part of the plasma and in the vicinity of zero of the electric field where the contribution from the sb-p regime to the non-ambipolar particle fluxes is expected to be largest. Drift-orbit resonances can contribute to the non-ambipolar particle fluxes nearly over whole the radial domain, except for a certain region in the vicinity of the zero of the electric field. For sufficiently large values of $m_{\vartheta}$ drift-orbit resonances occur not only in the deeply trapped region but also in the vicinity of the trapped-passing boundary. This emphasizes the importance of a proper numerical discretization of the velocity space in order to resolve the different resonant transport regimes.
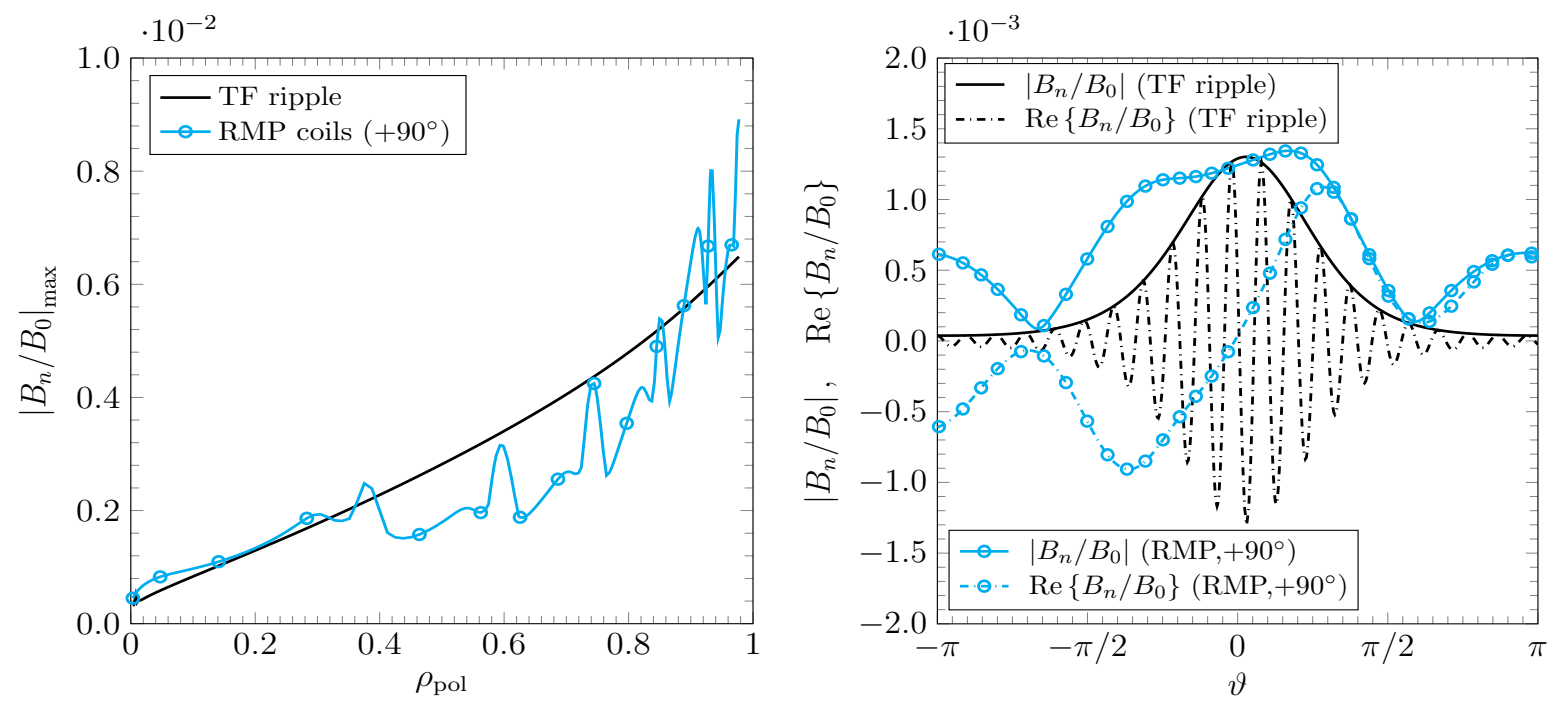

Figure 2. Left - radial profiles of the maximum value of the normalized perturbation field for the TF ripple and for RMP with $90^{\circ}$ coil phase (see definition in Eq. (14)); right - Variation of the absolute value and real part of the normalized perturbation field along the field line for $\rho_{\text {pol }}=0.5$.

For the TF ripple a comparison of NEO-2 results with analytical estimates of the torque density and of the integral torque,

$$
\left(T_{\varphi}^{\mathrm{NA}}\right)_{\text {int }}=\int_{V\left(\rho_{\mathrm{pol}}\right)} \mathrm{d}^{3} r T_{\varphi}^{\mathrm{NA}}
$$

where $V\left(\rho_{\text {pol }}\right)$ is the volume limited by the flux surface with a given $\rho_{\text {pol }}$, is shown in Figure 4. In order to quantify the impact of nonlinear effects and to validate the quasilinear approach, the NTV torque density is also computed with the code SFINCS. It can be seen that the NTV torque acts in the direction opposite to the experimentally measured plasma rotation velocity and the integral torque computed by NEO-2 is about $-0.6 \mathrm{Nm}$, whereas the SFINCS calculation predicts a value of $-0.4 \mathrm{Nm}$. The NTV torque produced by TF ripples is mainly applied to ions and, in case of the quasilinear model, corresponds to the ripple-plateau regime [10] in a major part of the plasma volume. The difference between the NEO-2 result and the SFINCS result is about $30 \%$ for $\rho_{\text {pol }} \leq 0.9$ and a rather large discrepancy is observed at outermost points with $\rho_{\text {pol }}>0.9$. This 

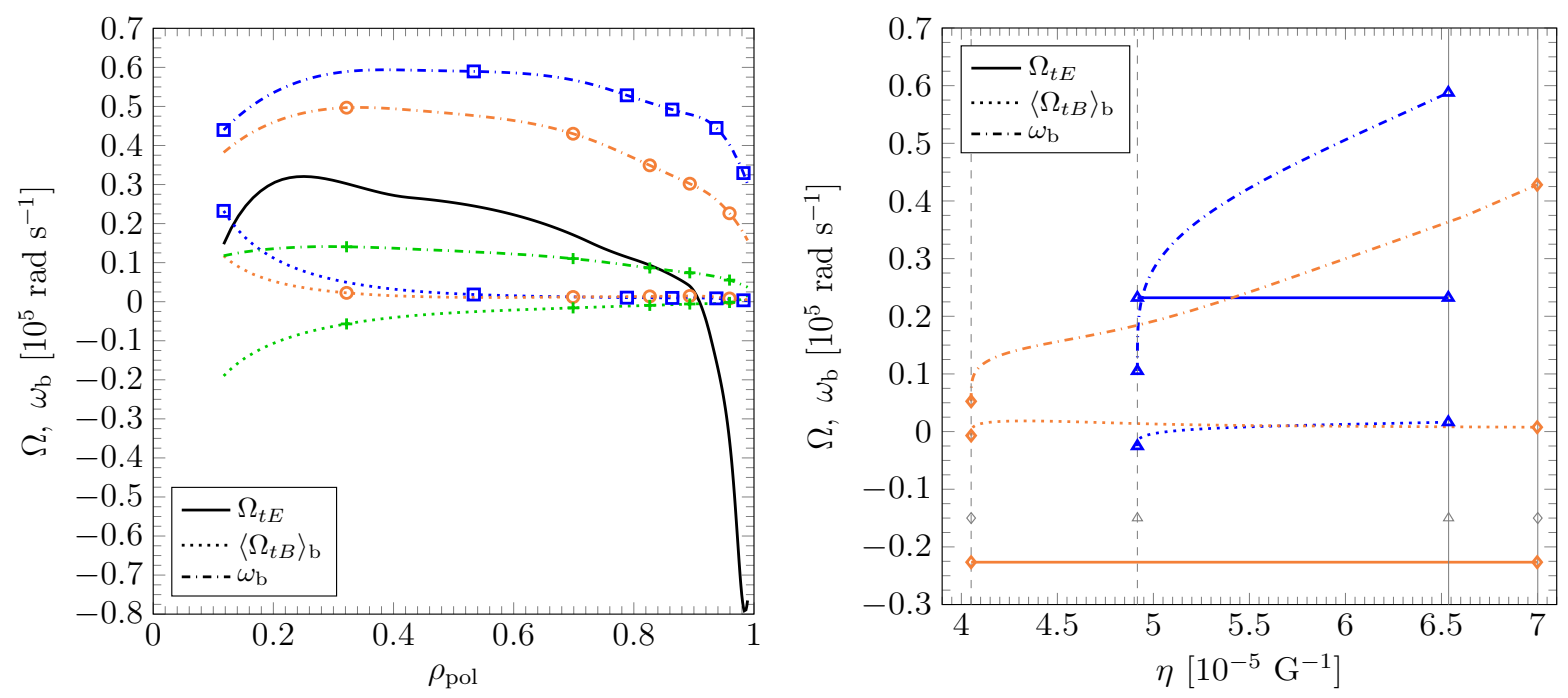

Figure 3. Left - radial profiles of the ion $\mathbf{E} \times \mathbf{B}$ drift frequency $\Omega_{t E}$, bounce-averaged magnetic drift frequency $\left\langle\Omega_{t B}\right\rangle_{\mathrm{b}}$ and bounce frequency $\omega_{\mathrm{b}}$ in the deeply trapped ( $\square$ ), trapped-passing boundary $(+)$ and an intermediate (o) region. Right - drift and bounce frequencies as functions of the normalized perpendicular adiabatic invariant $\eta$ at $\rho_{\text {pol }}=0.57(\triangle)$ and $\rho_{\text {pol }}=0.95(\diamond)$ for the trapped particle domain. The trappedpassing and deeply trapped boundaries are indicated by dashed and solid vertical lines, respectively.
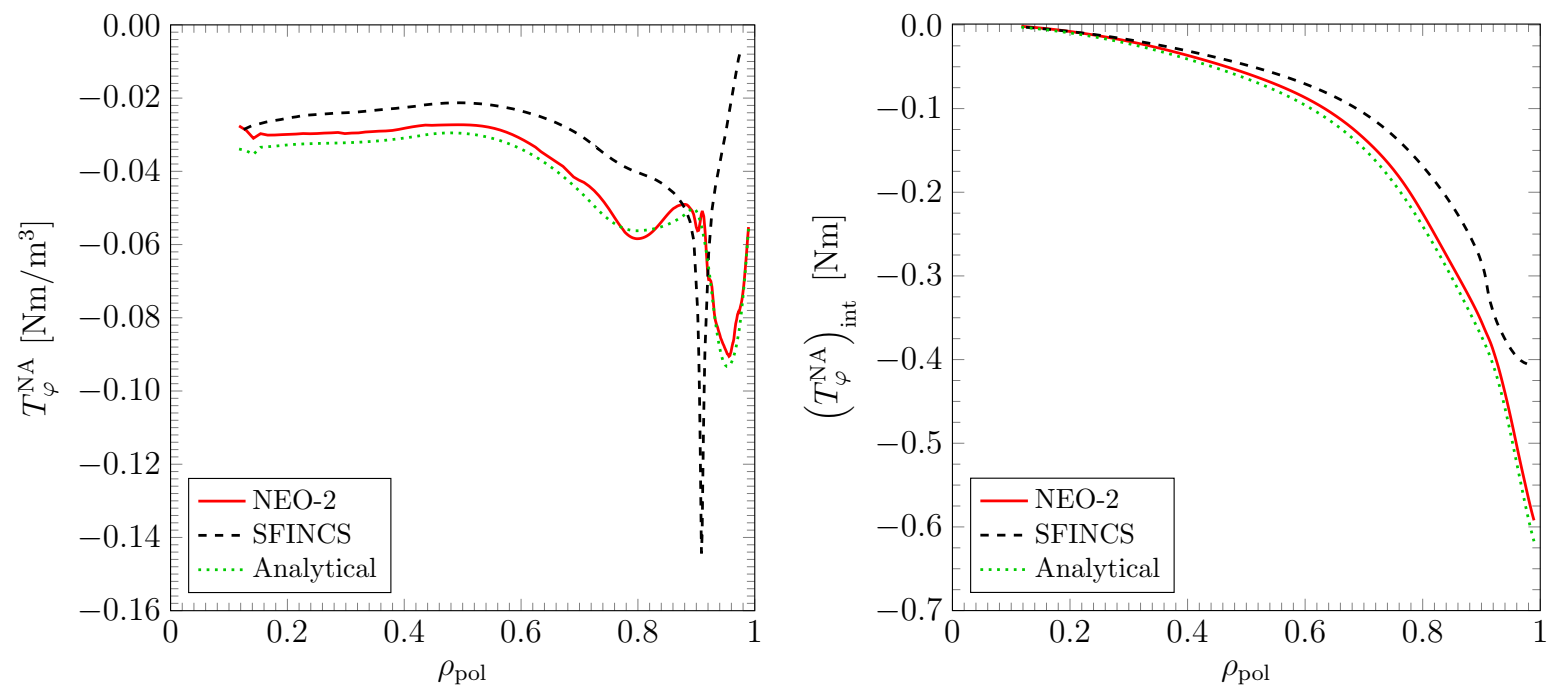

Figure 4. Radial profiles of the NTV torque density (left) and the integral torque (right) produced by the TF ripple.

correlates with the deviation from the quasilinear scaling of the NTV torque density, see Figure 5, and can be attributed to the onset of nonlinear transport due to locally trapped particles, which are blocked by the perturbation field. The respective quasilinear theory validity conditions (41) of Ref. [8] are clearly violated at outermost points and are marginally violated in the rest of the plasma volume. For the SFINCS calculation shown here, only the $\mathbf{E} \times \mathbf{B}$ drift of particles within flux surfaces has been taken into account. 


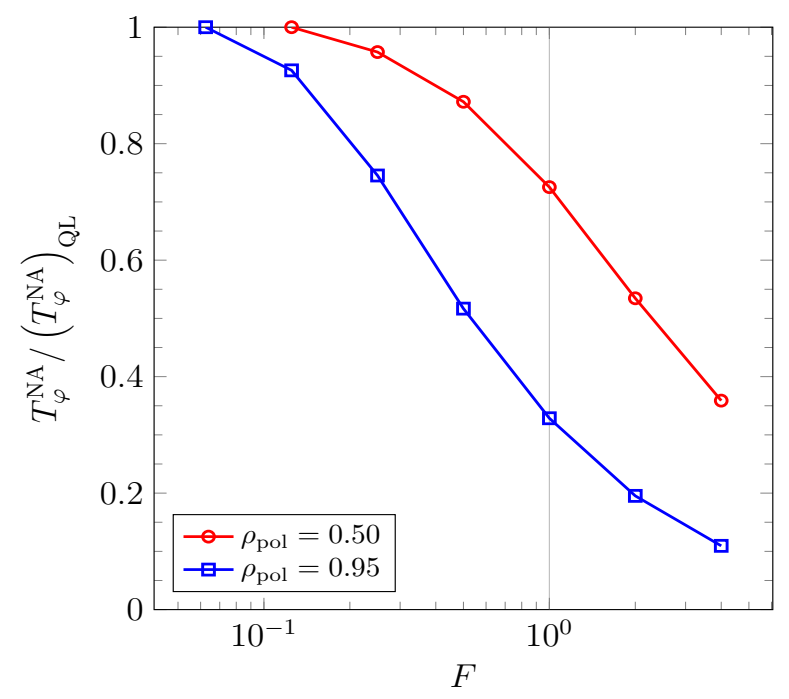

Figure 5. Ratio of the NTV torque density computed by SFINCS $T_{\varphi}^{N A}$ to the quasilinear result $\left(T_{\varphi}^{N A}\right)_{\mathrm{QL}}$ as a function of the perturbation field scaled by a factor $F$ for the TF ripple at different radial positions. $F=1$ corresponds to the actual value of the perturbation field in the experiment.

Therefore a strong increase of the torque is seen near the zero of radial electric field at $\rho_{\text {pol }} \approx 0.9$, where a contribution of $1 / \nu$ transport appears. This increase is absent in the NEO-2 result where also the magnetic drift is taken into account (see a more detailed discussion of the RMP case below). Due to these differences, the value of the integral torque computed by NEO-2 is about 33\% larger than the value predicted from SFINCS computations. The analytical estimate, used here for comparison, is obtained from a general expression for the particle flux in the ripple-plateau regime (Eq. (45) of Ref. [10]) by replacing in this derivation the simplified magnetic field with the more general form,

$$
B(r, \vartheta, \varphi)=B_{0}(r, \vartheta)+\operatorname{Re}\left\{B_{n}(r, \vartheta) \exp (i n \varphi)\right\},
$$

where $B_{0}$ is the unperturbed magnetic field and $B_{n}$ is a complex perturbation field amplitude. The non-ambipolar diffusion coefficients valid for a general tokamak geometry are then

$$
\begin{aligned}
& D_{11}^{\mathrm{NA}}=\frac{\sqrt{\pi}}{4} \frac{n m_{i}^{2} c^{2} v_{T}^{3} B_{0}^{2}}{e_{i}^{2} g\left(B_{0}^{\vartheta}\right)^{2} B_{0}^{\varphi}}\left(\int_{0}^{2 \pi} \frac{\mathrm{d} \vartheta}{B_{0}^{2}}\right)^{-1} \int_{0}^{2 \pi} \frac{\mathrm{d} \vartheta}{B_{0}^{3}}\left|\frac{B_{n}}{B_{0}}\right|^{2}, \\
& D_{12}^{\mathrm{NA}}=3 D_{11}^{\mathrm{NA}},
\end{aligned}
$$

where the notation is the same as in Ref. [8]. The difference in the integral torque between the NEO-2 result and the analytical estimate is less than $5 \%$.

In Figure 6, the NEO-2 result for the ion NTV torque density produced by the ELM mitigation coils with $\Delta \phi_{\mathrm{ul}}=90^{\circ}$ is compared to the bounce-averaged model of Shaing $[5,8]$ and, with the toroidal rotation due to the magnetic drift set to zero, also to a semi-analytical model based on a Hamiltonian approach [11] and to the code 
SFINCS. If only the $\mathbf{E} \times \mathbf{B}$ drift is considered, the agreement with SFINCS is nearly perfect (blocked particles are absent for medium scale perturbations), and there is a qualitatively good agreement between NEO-2 and the bounce-averaged model [5] in the vicinity of the zero of the electric field around $\rho_{\text {pol }}=0.9$. Apart from this radial domain NEO-2 results exceed the results of the bounce-averaged model [5] significantly. The discrepancies can be fixed by adding the NTV torque density from resonant (not bounce-averaged) transport regimes, which is computed by the semi-analytical model [11] in the collisionless limit, to the bounce-averaged model [5]. In radial domains with sufficiently large $E_{r}$, where the contribution from drift-orbit resonances to the NTV torque is dominating, a very good agreement between the NEO-2 result and the semi-analytical model is found. For the NEO-2 result including both, $\mathbf{E} \times \mathbf{B}$ drift and magnetic drift, a modification of the NTV torque density is observed in the core of the plasma and in the vicinity of the zero of the electric field. This emphasizes the necessity to include the magnetic drift into the computations. It should be noted that the contribution of the magnetic drift to the canonical toroidal banana precession frequency obtained within the Hamiltonian approach of Ref. [11] differs from the result of bounce averaging of the rotation frequency $\Omega_{t B}$ given by Eq. (67) of Ref. [8] by the presence of an additional term proportional to $q^{\prime}$, i.e. to the magnetic shear. This is the result of using the standard neoclassical ansatz as a starting point for the derivation of quasilinear equations in Ref. [8]. In the standard neoclassical ansatz orbits used for the computation of a linear perturbation of the distribution function are local, bounded to a particular flux surface (see also Refs. [12, 17]). The semi-analytical model [11] taking into account drift-orbit and sb-p resonances agrees very well with the local NEO2 result over the whole radial domain, if the magnetic shear is neglected in that model. For $\rho_{\text {pol }}>0.7$ the sb-p resonance makes a dominant contribution to the NTV torque. A good agreement between the semi-analytical model considering only sb-p resonance and the bounce-averaged model [5] is found for $\rho_{\text {pol }}<0.2$. The discrepancies in the vicinity of the zero of the electric field are due to finite aspect ratio and deviations from the circular flux surface approximation.

The numerical approach implemented in the quasilinear version of the code NEO-2, which is based on a standard local neoclassical ansatz, can be extended to a non-local quasilinear NTV model. Being rather different in derivation, the nonlocal quasilinear equation set and expressions for the non-ambipolar particle flux density are formally the same with results of Ref. [8]. Only the toroidal rotation frequency due to the magnetic drift $\Omega_{t B}$ is modified which, instead of local Eq. (67) of Ref. [8], is now given by the following nonlocal expression,

$$
\begin{aligned}
\Omega_{t B}= & \frac{v^{2}\left(2-\eta B_{0}\right)}{2 \sqrt{g_{0}} B_{0} \omega_{c 0}}\left(\frac{B_{r}}{B_{0}} \frac{\partial B_{0}}{\partial \vartheta}-\frac{B_{0}}{B_{0}^{\vartheta}} \frac{\partial B_{0}}{\partial r}\right)+ \\
& +\frac{v^{2}\left(1-\eta B_{0}\right)}{\sqrt{g_{0}} B_{0} \omega_{c 0}}\left(\frac{\partial}{\partial r}\left(B_{\vartheta}+q B_{\varphi}\right)-\frac{\partial B_{r}}{\partial \vartheta}\right),
\end{aligned}
$$

where the only difference from the local expression is the presence of the safety factor under radial derivative sign. The extended formalism pertinent to the non-local NEO-2 
version will be presented in a separate publication. The additional magnetic shear term leads to a significant modification of the torque density profile, see Figure 7. Again a very good agreement between the non-local version of NEO-2 and the semianalytical model [11] can be observed, which indicates the importance of contributions from various resonant transport regimes to the ion NTV torque density. In comparison to the results obtained by the local approach the sb-p regime covers only a narrow radial domain located at the zero of the electric field. Furthermore, a distinctive peak of the torque density is observed at $\rho_{\text {pol }}=0.4$. It should be noted that the expression for the NTV torque density in the sb-p regime derived from the Hamiltonian approach agrees analytically with Shaing's generalized formula [26] published recently.

As can be seen from Figure 8, not only ions but also electrons make a significant contribution to the NTV torque, which is in the direction of (positive) plasma rotation and which partly balances the negative ion torque. In the case where only the $\mathbf{E} \times \mathbf{B}$ drift is taken into account, the electron torque agrees up to a factor 3 with the result of the asymptotical model [5]. The observed discrepancies can be explained by uncertainties in the joining procedure of the different asymptotical regimes and by the rather small aspect ratio where the analytical model of Shaing [5] can significantly deviate from accurate computations with NEO-2 [8]. It can be observed that the agreement becomes better for larger aspect ratios closer to the center of the plasma and for small values of the radial electric field where the contribution from pure $1 / \nu$ transport is dominant.

Including the magnetic drift term in the NEO-2 computation modifies significantly the electron NTV torque density profile in the core of the plasma. In the inner part of the plasma only the trend seen from NEO-2 results is captured by the universal formula [5] connecting the $1 / \nu, \nu-\sqrt{\nu}$ and sb-p transport regimes. The observed differences in the inner part of the plasma are due to the circular flux surface approximation used for the evaluation of the sb-p resonance condition, which can deviate significantly from accurate computations for a real tokamak geometry. It should also be noted that the torque density profile exhibits distinctive substructures in the vicinity of resonant surfaces, which are indicated by vertical lines. A rather peculiar point in this profile is the resonant surface $(m, n)=(6,2)$ which almost coincides with the zero of the electric field. The increased electron torque density around this point is due to the fact that for small values of the electric field $1 / \nu$ transport is dominant. The electron NTV torque density evaluated by the non-local version of NEO-2 differs considerably from local computations for $\rho_{\text {pol }}>0.3$. The additional magnetic shear term yields not only a modification of the sb-p transport at intermediate radii, but affects also the $1 / \nu$ and $\nu-\sqrt{\nu}$ transport at the edge.

The NTV torque densities shown in Figure 7 and Figure 8 can be also expressed in terms of slowing down frequencies $\nu_{\mathrm{s}}^{\alpha}$ and offset rotation frequencies $V_{\mathrm{eq}, \alpha}^{\varphi}$ via the generic form (see, e.g., (5) of Ref. [8]),

$$
T_{\varphi}^{\mathrm{NA}}=-n_{i} m_{i} \sum_{\alpha} \nu_{\mathrm{s}}^{\alpha}\left\langle g_{\varphi \varphi}\left(V^{\varphi}-V_{\mathrm{eq}, \alpha}^{\varphi}\right)\right\rangle=-n_{i} m_{i} \nu_{\mathrm{s}}^{i+e}\left\langle g_{\varphi \varphi}\left(V^{\varphi}-V_{\mathrm{eq}, i+e}^{\varphi}\right)\right\rangle,(17
$$

which provides a rather demonstrative representation. Here, $g_{\varphi \varphi}=R_{0}^{2}$ denotes the 
toroidal covariant metric tensor component. In Figure 9 the respective radial profiles of the species offset rotation frequencies evaluated by the non-local version of NEO-2 are shown. It can be seen that ions tend to rotate in the negative toroidal direction, whereas the offset rotation frequency of electrons is positive. The different sign of the offset rotation frequency of ions and electrons correlates with the sign of the torque density. The total offset rotation frequency exhibits a rather remarkable behavior at intermediate radial positions where it oscillates around the measured toroidal rotation frequency. At the edge the computed total offset rotation frequency deviates significantly from the measured value.
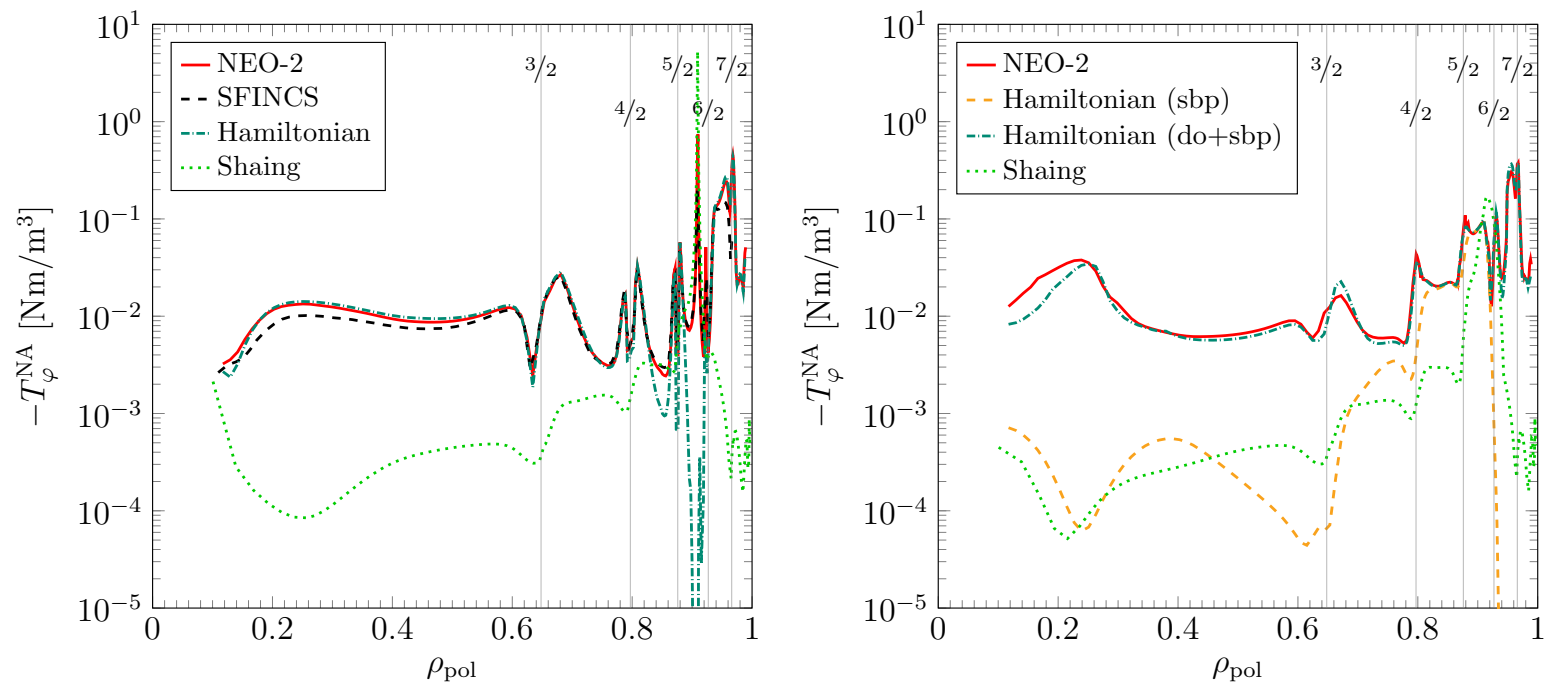

Figure 6. Ion contribution to the NTV torque density produced by the ELM mitigation coils with a phase of 90 degrees as a function of the normalized poloidal radius. Neglecting the effect of magnetic drift (left), the NEO-2 result (solid line) is compared to SFINCS (dashed line), to a semi-analytical Hamiltonian model [11] (dashdotted line) and to the bounce-averaged model by Shaing $[5,8]$ (dotted line). The NEO-2 result including both, $\mathbf{E} \times \mathbf{B}$ drift and magnetic drift, is compared to the semianalytical Hamiltonian model taking into account drift-orbit (do) and superbananaplateau (sbp) resonances, as well as to the universal formula [5] connecting $1 / \nu$, $\nu-\sqrt{\nu}$ and superbanana-plateau transport regimes (right). Vertical lines indicate the positions of resonant surfaces with $q\left(\rho_{\text {pol }}\right)=m / n$, where $m$ and $n$ are the poloidal and toroidal mode numbers, respectively.

In order to determine relevant transport regimes, a scan of the diffusion coefficient $D_{11}^{\mathrm{NA}}$ normalized by the plateau diffusion coefficient $D_{p}$ over collisionality parameter and otherwise the same parameters as in the experimental profile has been performed at different radial positions, see Figure 10. Here, $D_{p}=\pi q v_{T} \rho_{L}^{2}\left(16 R_{0}\right)^{-1}, \rho_{L}=v_{T} / \omega_{c 0}$, $\omega_{c 0}$ is the mean cyclotron frequency value at a given flux surface, and only the dominant $n=2$ perturbation toroidal mode has been taken into account. For electrons all quasilinear transport regimes described by the bounce-averaged drift kinetic equation can be seen. At $\rho_{\text {pol }}=0.30$ electrons are clearly in a transition regime between the $1 / \nu$ and the superbanana-plateau regime. At $\rho_{\text {pol }}=0.50$ the onset of the $\nu-\sqrt{\nu}$ regime 


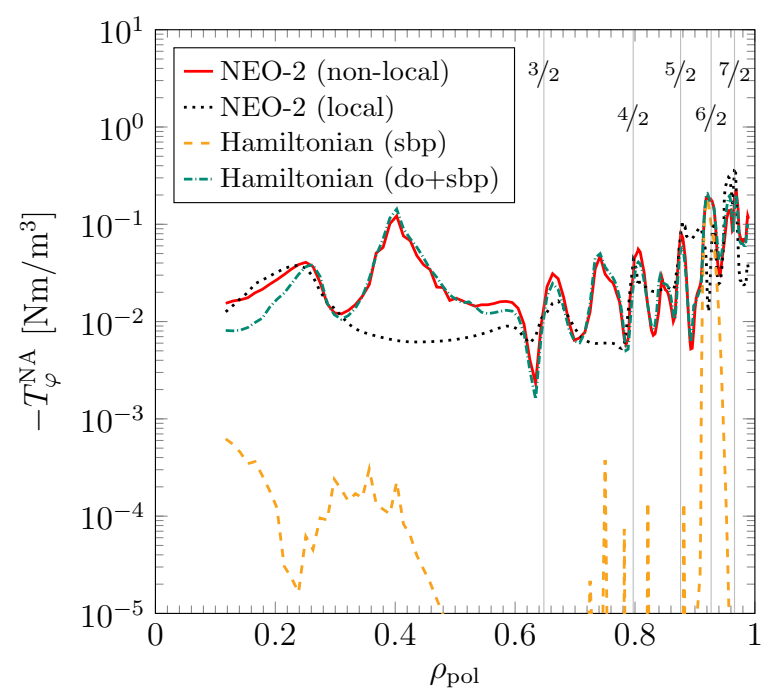

Figure 7. Ion contribution to the NTV torque density produced by the ELM mitigation coils with a phase of 90 degrees as a function of the normalized poloidal radius. The non-local NEO-2 result including both, $\mathbf{E} \times \mathbf{B}$ drift and magnetic drift, is compared to the NEO-2 result using a local approximation and to the semianalytical Hamiltonian model taking into account drift-orbit (do) and superbananaplateau (sbp) resonances. Vertical lines indicate the positions of resonant surfaces with $q\left(\rho_{\text {pol }}\right)=m / n$, where $m$ and $n$ are the poloidal and toroidal mode numbers, respectively.

is observed, whereas at $\rho_{\text {pol }}=0.91$ electrons are still in the $1 / \nu$ regime and only at lower collisionalities the transition to the superbanana plateau is observed. When the magnetic shear is taken into account in the non-local computations, the sb-p regime seen at $\rho_{\text {pol }}=0.91$ is replaced by a $\nu-\sqrt{\nu}$ regime. For other radii the computations with and without magnetic shear differ only slightly. It should be noted that the difference in the torque density seen in Figure 8 at $\rho_{\text {pol }}=0.50$ is due to the contribution from the $n=6$ perturbation, which is not considered here. The plateau like behavior of the ion diffusion coefficient indicates resonant "collisionless" diffusion regimes because the ion collisionality for $n=2$ perturbations stays outside the lower boundary of the ripple plateau regime [10], which requires $\nu^{*}>(n q)^{-2} A^{-3 / 2}$ with $A$ being the aspect ratio. The non-local results qualitatively show the same dependence on collisionality, although the absolute value of the resonant regime can deviate significantly. These regimes are of different nature for different radii. For $\rho_{\text {pol }}=0.3$ and $\rho_{\text {pol }}=0.5$ where Mach numbers are rather large, the resonant regime corresponds to the regime of bounce resonances $[27,16]$. It can be seen from Figure 6 that toroidal rotation due to the magnetic drift starts to be important for bounce resonances at smaller $\rho_{\text {pol }}$, in particular at $\rho_{\mathrm{pol}}=0.3$, since the frequency of this rotation scales inversely with $\rho_{\mathrm{pol}}$. In contrast to the two inner points in Figure 10, the ion diffusion coefficient at $\rho_{\mathrm{pol}}=0.91$, which is close to $E_{r}=0$ point, corresponds to the superbanana plateau regime. Its value there is much higher than the ripple plateau value, which is roughly the maximum value achievable in the regime of bounce resonances (see [11]). This can also be seen from 

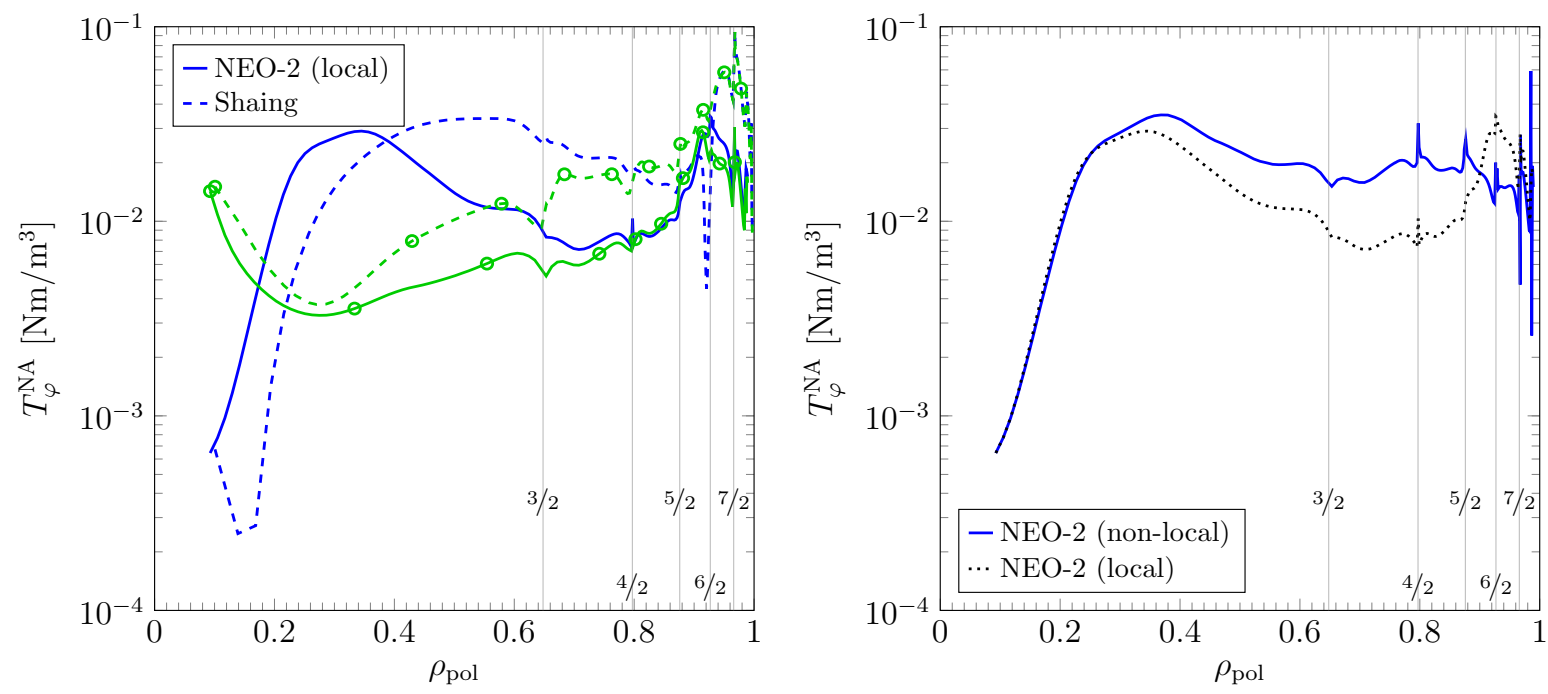

Figure 8. Electron contribution to the NTV torque density produced by the ELM mitigation coils with a phase of 90 degrees as a function of the normalized poloidal radius. Left - the NEO-2 results (solid lines) including magnetic drift (without markers) and neglecting magnetic drift (with markers) are compared to the bounce-averaged model by Shaing [5] (dashed lines) for the same cases. Right - the non-local NEO-2 result including both, $\mathbf{E} \times \mathbf{B}$ drift and magnetic drift, is compared to the NEO-2 result using a local approximation. Vertical lines indicate the positions of resonant surfaces with $q\left(\rho_{\text {pol }}\right)=m / n$, where $m$ and $n$ are the poloidal and toroidal mode numbers, respectively.

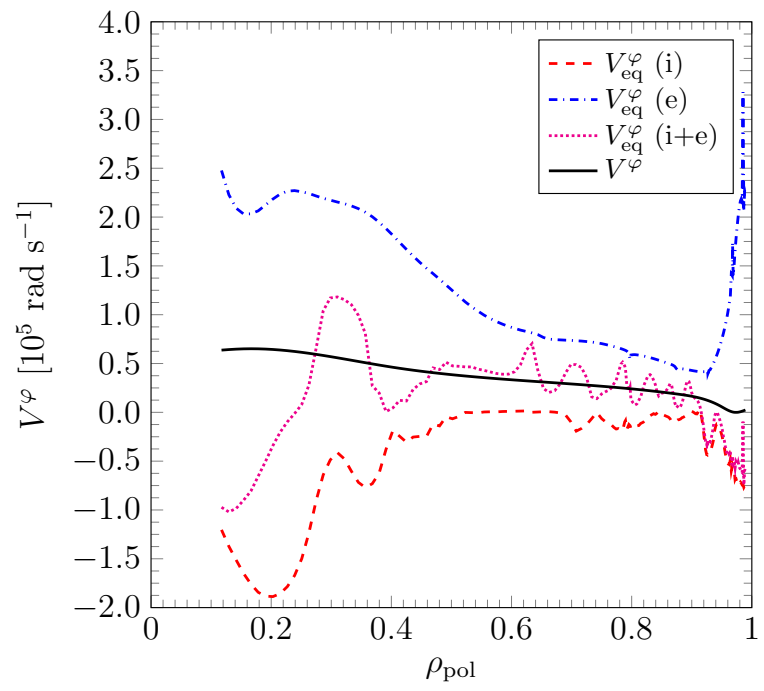

Figure 9. Non-local NEO-2 result for the offset rotation frequencies (17) produced by the ELM mitigation coils with a phase of 90 degrees as a function of the normalized poloidal radius for ions (dashed), electrons (dash-dotted) and sum over species (dotted). For comparison the radial profile of the measured toroidal rotation frequency (solid line) is shown.

Figure 6 , where at $E_{r}=0$ a spike of the torque appears in case of a pure $\mathbf{E} \times \mathbf{B}$ rotation because of a strong $1 / \nu$ contribution. This is changed to a saturated value when the 
complete rotation model is used.

As seen in Figure 11 the integral NTV torque $\left(T_{\varphi}^{\mathrm{NA}}\right)_{\text {int }}$, Eq. (13), is dominated by the ion contribution and is produced mainly at the plasma edge. The maximum value of the total integral NTV torque as computed by NEO-2 is $-1 \mathrm{Nm}$, which is less than the NBI torque value of $+4.1 \mathrm{Nm}$. As can be seen from the gradients of the integral torque, the NTV torque density is larger than the one from NBI at $\rho_{\text {pol }}>0.9$ where NTV dominates above NBI in the formation of the toroidal rotation profile.

In the torque from RMP coils electrons play an equally important role as ions and may even dominate in the plasma core for some coil polarities making the torque in the core positive (see Figure 12). In turn, for $\rho_{\text {pol }}>0.8$ ions are always dominant, and finally the ions determine the sign of the total torque from RMP coils in all cases. The magnitude of NTV torque produced by the RMP coils depends strongly on the poloidal field spectrum. The largest values of the integral NTV torque can be seen for $\Delta \phi_{\mathrm{ul}}=+90^{\circ}$ and $\Delta \phi_{\mathrm{ul}}=-150^{\circ}$. In case of $\Delta \phi_{\mathrm{ul}}=+30^{\circ}$ and $\Delta \phi_{\mathrm{ul}}=+52^{\circ}$ the smallest electron contribution to the NTV torque is observed, whereas for negative values of the phase shift the electron NTV torque is considerably increased. The effect of magnetic shear, which is taken into account by the non-local computations, is largest for negative coil polarities and for $\Delta \phi_{\mathrm{ul}}=+90^{\circ}$, whereas $\Delta \phi_{\mathrm{ul}}=+30^{\circ}$ and $\Delta \phi_{\mathrm{ul}}=+52^{\circ}$ are unaffected to a large extent. Here, ions dominate the integral NTV torque for $\rho_{\text {pol }}>0.4$, which is due to the additional peak in the ion torque density seen in Figure 7. A positive value of the torque in the core is only observed for negative coil polarities.

As seen in Figure 13, the magnitude of the RMP torque for various coil polarities correlates with the maximum value of the normalized perturbation field and with the maximum corrugation of flux surfaces (see Figure 4 in Ref. [20]) for the respective phases

(roughly $\left|B_{n} / B_{0}\right|_{\max } \sim\left|\delta_{\mathrm{N}}\right|_{\max } R_{0}^{-1}$ ). This means that the main reason for the nonaxisymmeric perturbation of the magnetic field magnitude $B$ on perturbed flux surfaces is the meandering of these surfaces caused by the perturbation field component normal to the unperturbed flux surfaces, but not the direct change of $B$ by the component which is parallel to the unperturbed field $[5,7,6]$.

\section{Integral momentum balance and other momentum sources}

It should be mentioned that plasma parameter profiles used in this modelling, in particular the toroidal rotation frequency, correspond to a (quasi-)steady state observed in shot \#30835 at 3.2 s. In contrast to Ref. [1] where a modification of a steady state rotation profile by turning on perturbation coils has been shown to be in agreement with NTV induced by these coils, this can be hardly expected in the pertinent AUG shot. In this shot a significant density reduction and modification of temperature profiles has been observed after turning on the RMP coils, what leads also to a modification of turbulent transport. Therefore, a single steady state has been chosen for a study of the static torque balance. Since the integral rotational moment is a conserved quantity, the missing balance between the NTV torque and the NBI torque, which exceeds the NTV 

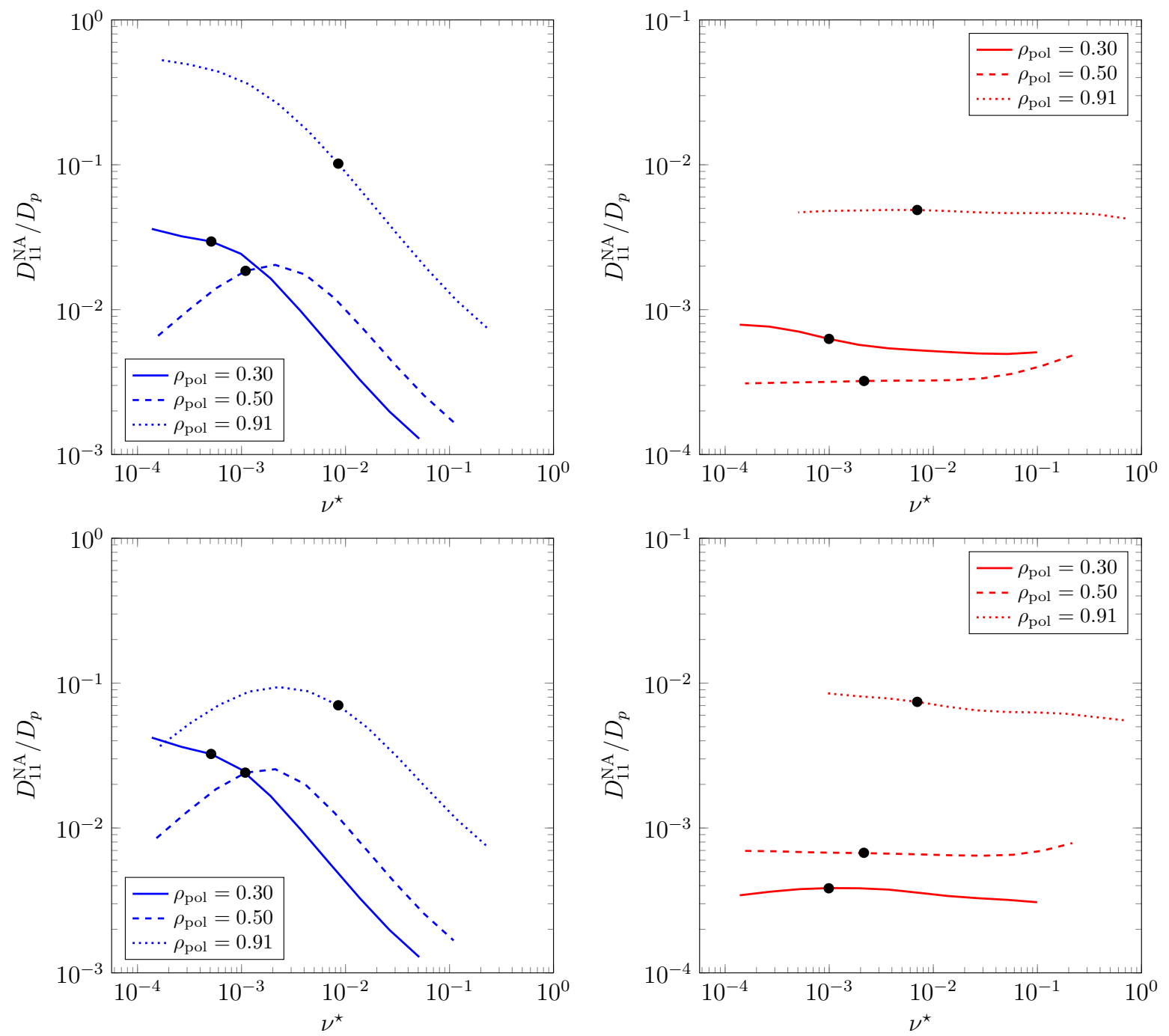

Figure 10. Normalized electron (left panel) and ion (right panel) diffusion coefficients $D_{11}^{\mathrm{NA}}$ computed with (lower panel) and without (upper panel) magnetic shear for various radii $\rho_{\text {pol }}$ (see the legend) as functions of the collisionality parameter $\nu^{\star}$. Actual collisionalities in the experiment for the given poloidal radius are indicated by filled circles. Note that the normalizing plateau diffusion coefficient is by a a square-root of mass ratio smaller for electrons than for ions.

torque roughly by a factor four, clearly suggests the importance of other momentum sources. The complete integral torque balance follows from the integration of the steady state equation (7) over the main plasma volume,

$$
\left(T_{\varphi}^{\mathrm{NBI}}\right)_{\mathrm{int}}+\left(T_{\varphi}^{\mathrm{NA}}\right)_{\mathrm{int}}+\left(T_{\varphi}^{\mathrm{tot}}\right)_{\mathrm{w}}=0
$$

where $\left(T_{\varphi}^{\mathrm{tot}}\right)_{\mathrm{w}}$ is the momentum flux through the main plasma boundary (separatrix) via $\left\langle\Pi_{[\mathrm{in}] \varphi}^{r}\right\rangle$, the only momentum source where the anomalous and axisymmetric neoclassical transport provide a contribution. Note that at least formally, all three torques in this balance can be determined independently outside the main plasma volume [28]. Since each of the last two torques in (18) consists of a few contributions, they are discussed 


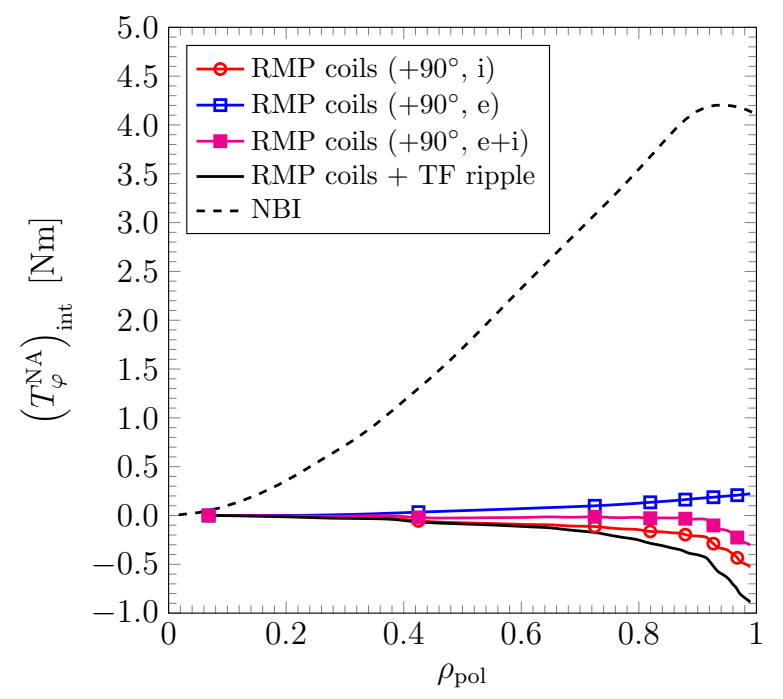

Figure 11. Profiles of various contributions to the integral NTV torque $\left(T_{\varphi}^{\mathrm{NA}}\right)_{\text {int }}$ for $90^{\circ}$ ELM mitigation coil polarity and of the integral NBI torque (see legend).
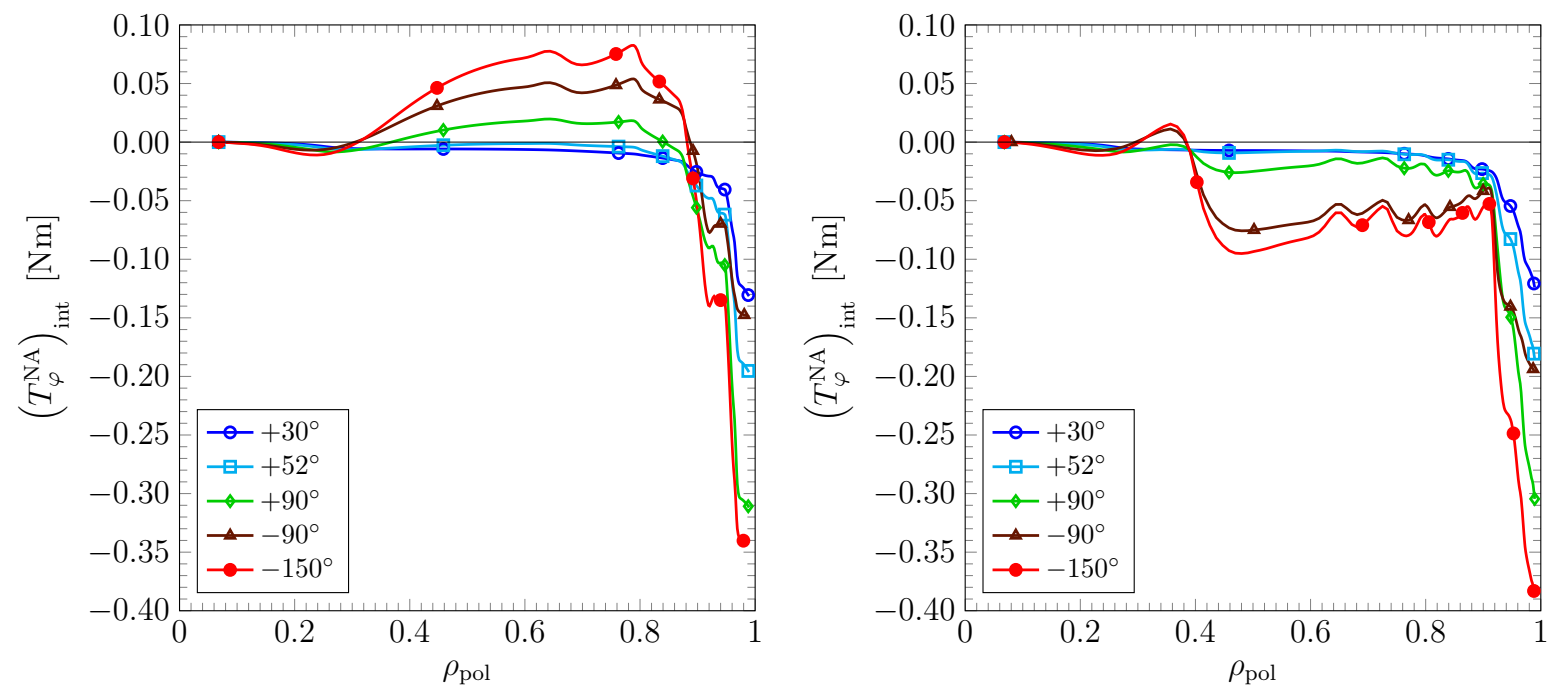

Figure 12. Profiles of total $(i+e)$ integral NTV torque from ELM mitigation coils with various polarities (see legend) for local (left) and non-local (right) computations.

below in more detail.

The contributions entering $\left(T_{\varphi}^{\mathrm{NA}}\right)_{\text {int }}$ besides the NTV torque are listed here roughly in the order of their importance. The first of these sources is related to losses of NBI generated fast particles $[29,30]$. This torque would be negative (as the NTV torque of ions) and its value can be high enough to balance the NBI torque, as shown in Ref. [30] for JET. Estimations of fast particle losses induced by violations of AUG axial symmetry using 3D Monte Carlo modelling in Boozer coordinates with help of the NEOMC code [31] version for fast particles [32] have shown that the toroidal torque due to such losses cannot match the discrepancy because it is smaller than the NTV torque (about $5 \%$ of the NBI torque). Another important unaccounted momentum source is 

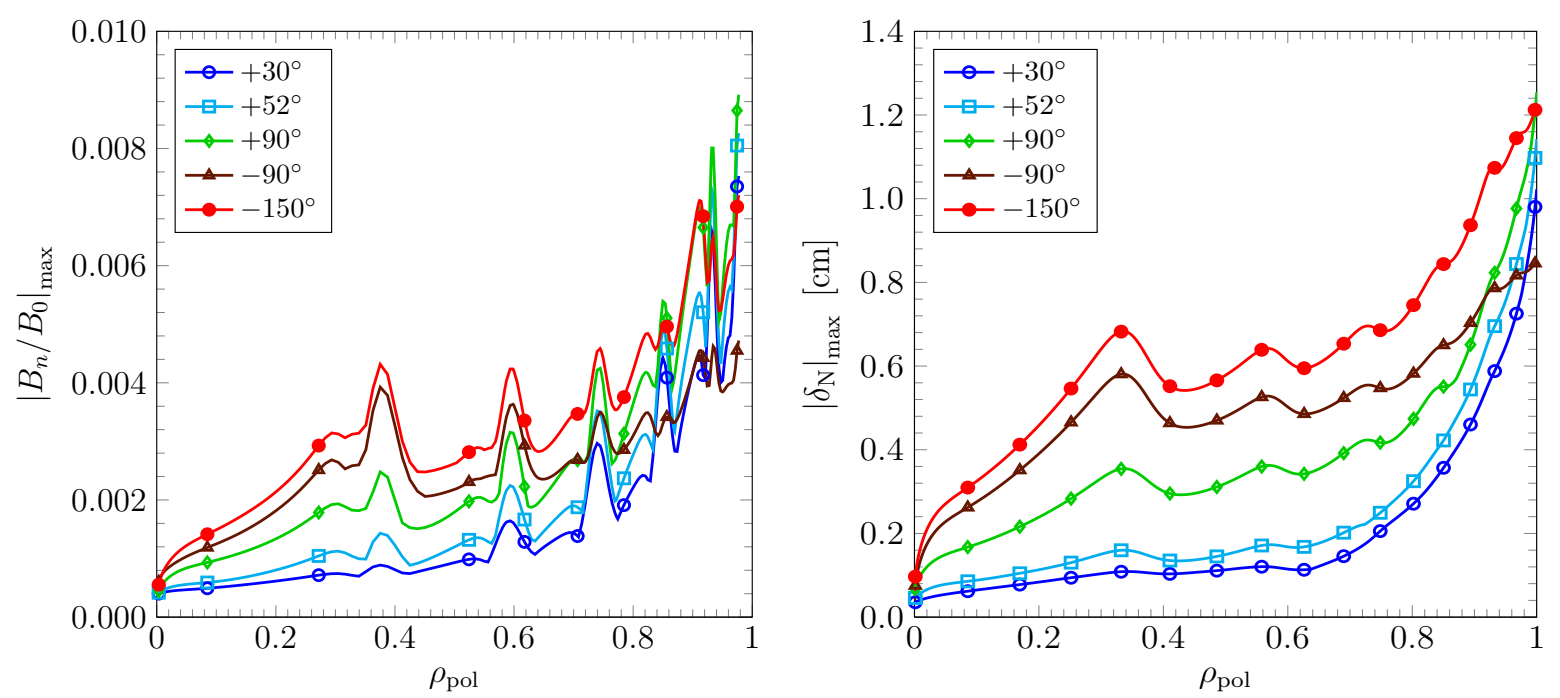

Figure 13. Profiles of the maximum value of the normalized perturbation field (left) and of the maximum corrugation of the 3D flux surface [20] (right) for ELM mitigation coils with various polarities (see legend).

the resonant torque produced by RMPs in resonant layers around rational flux surfaces where the ideal MHD theory is not valid. Normally, RMPs are shielded by plasma response currents in the core, which results in a small torque. However, the situation might change at the plasma edge, where the RMP amplitudes can be large enough to modify the electron temperature profile in the resonant layer around some of the dominating resonances. This might significantly reduce the shielding by electrons [21]. An accurate quantitative description of this interaction is still missing. Besides the magnetic perturbations from the TF ripple and ELM mitigation coils there are two more possible magnetic perturbations, which can produce NTV and resonant torque and which are not taken into account here. These are the error fields and the fields from eddy currents induced in the wall and other external conductors by intrinsic MHD modes.

It should be noted that a description of the resonant torque in terms of a local torque density $T_{\varphi}^{\mathrm{NA}}$ is limited to cases where the non-axisymmetric part of gradients of plasma parameters is small everywhere including the islands produced by RMPs. This is the case where the quasilinear theory is valid (see, e.g., [21]). Otherwise, this description is only formally valid, but not useful, because plasma parameters become essentially 3D within and in some vicinity of islands, and only the integral torque is meaningful.

The last contributor to $\left(T_{\varphi}^{\mathrm{NA}}\right)_{\text {int }}$, the torque due to ECRH/ECCD, consists of two parts. First, the direct toroidal momentum input by microwave radiatiation is related to the coupled ECRH power as $P_{\mathrm{ECRH}} R_{0} / c$, and for $P_{\mathrm{ECRH}} \sim 0.5 \mathrm{MW}$ is around $0.01 \mathrm{Nm}$. Second, losses of supra-thermal electrons, which are possible in presence of non-axisymmeric magnetic field perturbations, can produce only positive torque in the direction of the NBI torque. Therefore, the last contributor to $\left(T_{\varphi}^{\mathrm{NA}}\right)_{\text {int }}$, the torque due to $\mathrm{ECRH} / \mathrm{ECCD}$, can be safely removed from the list. 
Various contributions to the torque $\left(T_{\varphi}^{\mathrm{NA}}\right)_{\text {int }}$ can be measured externally in a direct way because the integral torque due to electromagnetic perturbations is equal to the momentum flux carried by these perturbations through the plasma boundary in the form of Maxwell stress, essentially via its magnetic part. (Finally, this Maxwell stress is balanced by Lorentz forces onto the external currents that create the perturbations.) Therefore, it would be sufficient to determine the magnetic Maxwell stress by measuring the non-axisymmetric magnetic field outside the plasma [33, 28]. Alternatively, $\left(T_{\varphi}^{\mathrm{NA}}\right)_{\mathrm{int}}$ can be determined from the torque balance (18) using the measured $\left(T_{\varphi}^{\mathrm{NBI}}\right)_{\mathrm{int}}$ and $\left(T_{\varphi}^{\text {tot }}\right)_{\mathrm{w}}$. In absence of significant NBI and non-axisymmetric torque outside the main plasma boundary, the momentum flux $\left(T_{\varphi}^{\mathrm{tot}}\right)_{\mathrm{w}}$ carried through the separatrix is finally recovered at the wall, as follows from the integration of equation (1) over the vacuum chamber volume. Flux $\left(T_{\varphi}^{\text {tot }}\right)_{\mathrm{w}}$ consists of three contributions, which require different measurements. Presenting it as a sum of particle and electromagnetic field contributions, $\left(T_{\varphi}^{\mathrm{tot}}\right)_{\mathrm{w}}=\left(T_{\varphi}^{\mathrm{part}}\right)_{\mathrm{w}}+\left(T_{\varphi}^{\mathrm{EM}}\right)_{\mathrm{w}}$ and averaging this expression over the time scale of turbulent fluctuations, the toroidal reactive torque onto the main plasma due to charged and neutral particle fluxes to the wall is

$$
\left(T_{\varphi}^{\mathrm{part}}\right)_{\mathrm{w}}=2 \pi \sum_{\alpha} m_{\alpha} \oint \mathrm{d} l R \int \mathrm{d}^{3} v f_{\alpha} \mathbf{v} \cdot \mathbf{n} \mathbf{v} \cdot \frac{\partial \mathbf{r}}{\partial \varphi}
$$

where $f_{\alpha}$ is the averaged distribution function, which is then axisymmetric. The poloidal integration contour here is along the wall surface, and the unit vector normal to the wall $\mathbf{n}$ points to the inside of the vessel. In contrast to the momentum flux of neutral particles $(\alpha=\mathrm{n})$, which appears due to charge exchange with the surrounding neutral gas, and which is spread over the wall, the momentum flux of charged particles (ions) is localized at the divertor target plates. Before the sheath this latter flux is determined mainly by parallel transport, $\mathbf{v} \cdot \mathbf{n} \mathbf{v} \cdot \partial \mathbf{r} / \partial \varphi \rightarrow\left(v_{\|} / B\right)^{2} \mathbf{B} \cdot \mathbf{n} \mathbf{B} \cdot \partial \mathbf{r} / \partial \varphi$. This means that the toroidal reactive torque on the plasma from the vicinity of a particular strike point scales with $\mathbf{B} \cdot \mathbf{n}$. Since $\mathbf{B} \cdot \mathbf{n}$ has opposite signs for inner and outer strike points, the integral torque onto the main plasma manifests itself as an imbalance between the toroidal momentum fluxes carried by particles to the inner and outer divertor targets (see, e.g., Section 6.3 of Ref. [34] and Ref. [28]).

A simple expression for the intrinsic electromagnetic torque $\left(T_{\varphi}^{\mathrm{EM}}\right)_{\mathrm{w}}$, which has been omitted in Ref. [28], is obtained when the currents and charges induced by edge instabilities in the wall are negligible. In this case, only the axisymmetric part of the electromagnetic field resulting from averaging of this field over the fluctuation time scale contributes to the electromagnetic momentum flux, because only the axisymmetric field leads to a Lorentz force onto the axisymmetric currents in the wall. In a (quasi) steady state, the pertinent part of the Maxwell stress tensor $\boldsymbol{\sigma}$ is essentially determined by the magnetic field,

$$
\frac{1}{\sqrt{g}} \frac{\partial}{\partial x^{i}} \sqrt{g} \sigma_{\varphi}^{i}=\frac{1}{4 \pi} \nabla \cdot B_{\varphi} \mathbf{B}=\frac{1}{c} \nabla \cdot \psi_{\mathrm{pol}} \mathbf{j} .
$$


The integral intrinsic electromagnetic torque is then

$$
\begin{aligned}
\left(T_{\varphi}^{\mathrm{EM}}\right)_{\mathrm{w}} & =\frac{2 \pi}{c} \oint \mathrm{d} l R\left(\psi_{\mathrm{pol}}^{a}-\psi_{\mathrm{pol}}\right) \mathbf{j} \cdot \mathbf{n} \\
& \approx \frac{2 \pi}{c} \sum_{s} R_{s}^{2}(\mathbf{n} \cdot \mathbf{B})_{s} \int_{l_{s}-\Delta l}^{l_{s}+\Delta l} \mathrm{~d} l\left(l-l_{s}\right) \mathbf{j} \cdot \mathbf{n},
\end{aligned}
$$

where the final expression corresponds to the current localized around divertor strike points numbered here with subscript $s$. The term with $\psi_{\text {pol }}^{a}$ in the first expression in (21) provides a zero contribution to the integral due to $\nabla \cdot \mathbf{j}=0$. It should be noted that due to $(20)$ and $\nabla \cdot \mathbf{j}=0$, currents flowing along $\psi_{\text {pol }}$ contours (in particular, parallel currents) produce no steady state toroidal torque since they produce no toroidal $\mathbf{j} \times \mathbf{B}$ force. Only currents which are closed across $\psi_{\text {pol }}$ contours produce toroidal torque. This relates to both the currents in the plasma and to the poloidal currents in the wall, which close the plasma currents to the wall. The former produces the torque onto the plasma and the latter the torque onto the wall, thus balancing the force onto the plasma by third Newton's law. In the equilibria studied here, $\psi_{\text {pol }}$ increases from the private flux region towards the scrape-off layer. Thus, currents in the wall (target plates), which balance the positive NBI torque, flow in direction of increasing $\psi_{\text {pol }}$.

\section{Summary}

Computations of the NTV torque produced in ASDEX Upgrade by the TF ripple and magnetic perturbations from ELM mitigation coils show an agreement between different numerical (NEO-2, SFINCS) and semi-analytical models within their validity domains. Specific differences are also observed and discussed. It is clearly seen that ions as well as electrons contribute to the overall torque. It is remarkable that practically all quasilinear transport regimes except for the highly collisional Pfirsch-Schlüter regime are realized within the single discharge \#30835, which is studied here. Various bounce averaged transport regimes as well as resonant regimes are important in specific radial positions. Those regimes have been identified by scans over collisionality and comparison with analytical and semianalytical computations. The quasilinear approach used in NEO-2 is well justified for computations of the torque driven by RMPs but slightly overestimates the torque from the TF ripple. The amplitude of the perturbations corresponding to the TF ripple is already marginally outside the validity range for the quasilinear approach, mainly because of the high toroidal mode number.

The NTV torque is produced mainly at the plasma edge where its density is comparable with the NBI torque density. However, the integral NTV torque balances only a quarter of the integral NBI torque. This emphasizes the importance of other momentum sources unaccounted here. Some of these sources (e.g. the torque due to fast particle losses) can be computed with present day models. An accurate description of the other sources connected with resonant interaction of magnetic perturbations at 
rational flux surfaces is still an open problem. Thus, measurements of discharges where the role of resonant interactions is minimized are of future interest.

The integral torque balance [28], which can be used to verify the modelling by measurements outside the plasma, is discussed here in some more detail. For this balance, in addition to measurements of the asymmetry of the momentum flux carried by divertor fluxes [28], measurements of charge-exchange neutral spectra and of the currents between the plasma and the wall (divertor target plates) are shown to be of importance.

Besides the integral torque balance, an accurate description of the torque density profile resulting from non-axisymmetric magnetic field perturbations would be an important part of turbulent momentum transport studies, where the effect of turbulent

momentum flux dominating in $\left\langle\Pi_{[\mathrm{in}] \varphi}^{r}\right\rangle$ in equation (7) on the rotation velocity profile is required in its pure form, i.e. the second term in l.h.s. of (7). The state of art of existing models discussed here does already allow for such a description for the NTV torque.

\section{Acknowledgments}

This work has been carried out within the framework of the EUROfusion Consortium and has received funding from the Euratom research and training programme 20142018 under grant agreement No 633053. The views and opinions expressed herein do not necessarily reflect those of the European Commission. The authors gratefully acknowledge support from NAWI Graz. The author A. F. Martitsch acknowledges financial support from the "Friedrich Schiedel Stiftung für Energietechnik" during the initial phase of this work.

\section{References}

[1] W. Zhu, S. A. Sabbagh, R. E. Bell, J. M. Bialek, M. G. Bell, B. P. LeBlanc, S. M. Kaye, F. M. Levinton, J. E. Menard, K. C. Shaing, A. C. Sontag, and H. Yuh. Observation of plasma toroidal-momentum dissipation by neoclassical toroidal viscosity. Phys. Rev. Lett., 96:225002, Jun 2006.

[2] A. M. Garofalo, W. M. Solomon, M. Lanctot, K. H. Burrell, J. C. DeBoo, J. S. deGrassie, G. L. Jackson, J.-K. Park, H. Reimerdes, M. J. Schaffer, and E. J. Strait. Plasma rotation driven by static nonresonant magnetic fields. Phys. Plasmas, 16(5):056119, 2009.

[3] Y. Sun, Y. Liang, K. C. Shaing, Y. Q. Liu, H. R. Koslowski, S. Jachmich, B. Alper, A. Alfier, O. Asunta, P. Buratti, G. Corrigan, E. Delabie, C. Giroud, M. P. Gryaznevich, D. Harting, T. Hender, E. Nardon, V. Naulin, V. Parail, T. Tala, C. Wiegmann, S. Wiesen, T. Zhang, and JET-EFDA contributors. Non-resonant magnetic braking on JET and TEXTOR. Nuclear Fusion, 52(8):083007, 2012.

[4] E. Lazzaro, R. J. Buttery, T. C. Hender, P. Zanca, R. Fitzpatrick, M. Bigi, T. Bolzonella, R. Coelho, M. DeBenedetti, S. Nowak, O. Sauter, M. Stamp, and Contributors to the EFDAJET work programme. Error field locked modes thresholds in rotating plasmas, anomalous braking and spin-up. Phys. Plasmas, 9(9):3906-3918, 2002. 
[5] K. C. Shaing, S. A. Sabbagh, and M. S. Chu. An approximate analytic expression for neoclassical toroidal plasma viscosity in tokamaks. Nuclear Fusion, 50(2):025022, 2010.

[6] J. Park, A. H. Boozer, and J. E. Menard. Nonambipolar transport by trapped particles in tokamaks. Phys. Rev. Letters, 102(6):065002, 2009.

[7] Y. Sun, Y. Liang, K. C. Shaing, H. R. Koslowski, C. Wiegmann, and T. Zhang. Neoclassical toroidal plasma viscosity torque in collisionless regimes in tokamaks. Phys. Rev. Letters, 105(14):145002, 2010.

[8] S. V. Kasilov, W. Kernbichler, A. F. Martitsch, H. Maassberg, and M. F. Heyn. Evaluation of the toroidal torque driven by external non-resonant non-axisymmetric magnetic field perturbations in a tokamak. Phys. Plasmas, 21(9):092506, 2014.

[9] W. Kernbichler, S. V. Kasilov, G. O. Leitold, V. V. Nemov, and K. Allmaier. Recent progress in NEO-2 - A code for neoclassical transport computations based on field line tracing. Plasma and Fusion Research, 3:S1061-1-S1061-4, 2008.

[10] A. H. Boozer. Enhanced transport in tokamaks due to toroidal ripple. Phys. Fluids, 23(11):22832290, 1980 .

[11] C. G. Albert, M. F. Heyn, S. V. Kasilov, W. Kernbichler, and A. F. Martitsch. Toroidal rotation in resonant regimes of tokamak plasmas due to non-axisymmetric perturbations in the actionangle formalism. In 42nd EPS Conference on Plasma Physics, volume 39E, page P1.183, Lisbon, Portugal, 2015. European Physical Society.

[12] M. Landreman, H. M. Smith, A. Mollén, and P. Helander. Comparison of particle trajectories and collision operators for collisional transport in nonaxisymmetric plasmas. Phys. Plasmas, 21(4):042503, 2014.

[13] K. C. Shaing. Magnetohydrodynamic-activity-induced toroidal momentum dissipation in collisionless regimes in tokamaks. Phys. Plasmas, 10(5):1443-1448, 2003.

[14] K. C. Shaing, P. Cahyna, M. Becoulet, J.-K. Park, S. A. Sabbagh, and M. S. Chu. Collisional boundary layer analysis for neoclassical toroidal plasma viscosity in tokamaks. Phys. Plasmas, 15(8):082506, 2008.

[15] K. C. Shaing, S. A. Sabbagh, and M. S. Chu. Neoclassical toroidal plasma viscosity in the superbanana plateau regime for tokamaks. Plasma. Phys. Contr. Fusion, 51(3):035009, 2009.

[16] K. C. Shaing, M. S. Chu, and S. A. Sabbagh. Eulerian approach to bounce-transit and drift resonance and neoclassical toroidal plasma viscosity in tokamaks. Plasma. Phys. Contr. Fusion, 51(5):075015, 2009.

[17] S. P. Hirshman, K. C. Shaing, W. I. van Rij, C. O. Beasley, Jr., and E. C. Crume, Jr. Plasma transport coefficients for nonsymmetric toroidal confinement systems. Phys. Fluids, 29(9):29512959, 1986.

[18] V. V. Nemov, S. V. Kasilov, W. Kernbichler, and M. F. Heyn. Evaluation of $1 / \nu$ neoclassical transport in stellarators. Phys. Plasmas, 6(12):4622-4632, 1999.

[19] S. P. Hirshman, W. I. van RIJ, and P. Merkel. Three-dimensional free boundary calculations using a spectral green's function method. Computer Physics Communications, 43(1):143 - 155, 1986.

[20] E. Strumberger, S. Günter, and C. Tichmann. MHD instabilities in 3D tokamaks. Nuclear Fusion, 54(6):064019, 2014.

[21] M. F. Heyn, I. B. Ivanov, S. V. Kasilov, W. Kernbichler, P. Leitner, V. V. Nemov, W. Suttrop, and ASDEX Upgrade Team. Quasilinear Modelling of RMP Interaction with a Tokamak Plasma: Application to ASDEX Upgrade ELM Mitigation Experiments. Nuclear Fusion, 54(6):064005, 2014.

[22] A. Pankin, D. McCune, R. Andre, G. Bateman, and A. Kritz. The tokamak monte carlo fast ion module NUBEAM in the national transport code collaboration library. Computer Physics Communications, 159(3):157 - 184, 2004.

[23] M. Honda, T. Takizuka, K. Tobita, G. Matsunaga, and A. Fukuyama. Alpha particle-driven toroidal rotation in burning plasmas. Nuclear Fusion, 51(7):073018, 2011.

[24] Ya. I. Kolesnichenko and Yu. V. Yakovenko. Alpha-particle-induced toroidal flows in tokamak 
reactor plasma. Fusion Technology, 18(12):597-605, 1990.

[25] W. Suttrop, O. Gruber, S. Günter, D. Hahn, A. Herrmann, M. Rott, T. Vierle, U. Seidel, M. Sempf, B. Streibl, E. Strumberger, D. Yadikin, O. Neubauer, B. Unterberg, E. Gaio, V. Toigo, and P. Brunsell. In-vessel saddle coils for MHD control in ASDEX upgrade. Fusion Engineering and Design, 84(26):290-294, 2009. Proceeding of the 25th Symposium on Fusion Technology(SOFT25).

[26] K. C. Shaing. Superbanana and superbanana plateau transport in finite aspect ratio tokamaks with broken symmetry. J. Plasma Physics, 81:905810203, 2015.

[27] P. N. Yushmanov. Dokl. Akad. Nauk SSSR, 266:1123-1127, 1982.

[28] V. D. Pustovitov. Integral torque balance in tokamaks. Nuclear Fusion, 51(1):013006, 2011.

[29] T. Kurki-Suonio, S. K. Sipilä, and J. A. Heikkinen. Active diagnostic of edge Er using neutralparticle analysers. Plasma Physics and Controlled Fusion, 42(5A):A277 - A282, 2000.

[30] T. Koskela, A. Snicker, S. Sipilä, A. Salmi, T. Johnson, C. Marchetto, M. Schneider, M. Romanelli, H. Weisen, and JET contributors. Improvement of neutron yield predictions in JET with ASCOT. In 42nd EPS Conference on Plasma Physics, volume 39E, page P2.106, Lisbon, Portugal, 2015. European Physical Society.

[31] K. Allmaier, S. V. Kasilov, W. Kernbichler, and G. O. Leitold. Variance reduction in computations of neoclassical transport in stellarators using a f method. Physics of Plasmas, 15(7), 2008.

[32] V. V. Nemov, S. V. Kasilov, and W. Kernbichler. Collisionless high energy particle losses in optimized stellarators calculated in real-space coordinates. Physics of Plasmas, 21(6), 2014.

[33] N. C. Logan, E. J. Strait, and H. Reimerdes. Measurement of the electromagnetic torque in rotating DIII-D plasmas. Plasma. Phys. Contr. Fusion, 52(4):045013, 2010.

[34] R. Schneider, X. , Bonnin, K. Borrass, D. P. Coster, H. Kastelewicz, D. Reiter, V. A. Rozhansky, and B. J. Braams. Plasma Edge Physics with B2-Eirene. Contrib. Plasma Phys., 46(1-2):3-191, 2006. 